\title{
Preparation of Fluorescent Carbon-Based Dots from Waste Tire Pyrolysis
}

\author{
Juliana B. Parra, ${ }^{\#, a}$ Karina C. Silva, ${ }^{\#, a}$ Pedro A. S. Valezin, ${ }^{\#, a}$ Raphael G. Martins, \\ Rodolfo R. Gomes, ${ }^{\# a}$ Rafael S. Pereira, ${ }^{\circledR b}$ Fernando M. de Melo, ${ }^{\circledR c}$ \\ Andreia de A. Morandim-Giannetti, ${ }^{a}$ Ronaldo G. dos Santos, ${ }^{a}$ \\ Luz Consuelo G. A. Panzarinia ${ }^{a}$ and Marcos M. Toyama ${ }^{\circledR *, a}$ \\ ${ }^{a}$ Departamento de Engenharia Química, Centro Universitário FEI, \\ 09850-901 São Bernardo do Campo-SP, Brazil \\ ${ }^{b}$ Centro de Engenharia, Modelagem e Ciências Sociais Aplicadas, Universidade Federal do ABC, \\ 09210-580 Santo André-SP, Brazil \\ 'Instituto de Química, Universidade de São Paulo, 05508-000 São Paulo-SP, Brazil
}

\begin{abstract}
The pyrolysis of waste tire has been proposed as an alternative route to its recycling producing residual oils that can be used as fuels. In this work, a physicochemical process to obtain carbonbased dots $(\mathrm{CBD})$ from carbon black $\left(\mathrm{CB}_{\mathrm{L}}\right)$ derivatives of tires is described. For this, the $\mathrm{CB}_{\mathrm{L}}$ was treated with different acids to produce $\mathrm{CB}_{\mathrm{L} 1}$ and $\mathrm{CB}_{\mathrm{L} 2}$ particles suspension. The $\mathrm{pH}$ of the suspension was adjusted to 5.6 to precipitate remaining impurities. Afterwards, the supernatant was ultra-centrifuged and the yellow pale solid, the purified CBD obtained by roto evaporation, was characterized by ultraviolet-visible spectrophotometer (UV-Vis), photoluminescence (PL) at 320-380 nm, high-resolution transmission electron microscopy (HRTEM), scanning electron microscopy (SEM), thermogravimetric analysis (TGA), energy-dispersive X-ray spectroscopy (EDX), Fourier transform infrared microscopy ( $\mu$ FTIR), and X-ray photoelectron spectroscopy (XPS). This material showed the typical excitation wavelength dependent emission and a PL quantum yield of $19.29 \%$ and was constituted by a well-defined spherical shaped and single sheets of "graphene quantum dots" with undefined shape, with potential applications in optoelectronic and fluorescence imaging devices.
\end{abstract}

Keywords: waste tire, pyrolysis, carbon-based dots, quantum-dots

\section{Introduction}

The accumulation of industrial, agricultural, and urban waste has substantially increased, while residues of tires are considered a major environmental issue due to the continuous and growing amount corresponding to approximately $50 \%$ of all products sold annually (around 1.5 billion tires). Another problem is the severe environmental damage caused by their inadequate disposal, thus affecting the environment, e.g., water contamination, emission of harmful pollutants such as $\mathrm{SO}_{2},(\mathrm{NO})_{\mathrm{x}}$, and several organic compounds. ${ }^{1,2}$

In this context, the pyrolysis, a thermal degradation process carried out under an inert atmosphere from moderate to high temperatures (normally $400-900{ }^{\circ} \mathrm{C}$ ) has

*e-mail: mtoyama@fei.edu.br

"These authors contributed equally to this work. proven to be a viable alternative to recycle such residues. This occurs as the product of the pyrolysis is rich in molecules originated from the cleavage of $\mathrm{C}-\mathrm{C}$ bonds yielding smaller molecules, enabling the attainment of several products such as fuels, alkylbenzenes, aromatic compounds, terpenes, etc. It is also possible to generate products of higher added value, e.g., limonene in cosmetic and food industries. On the other hand, tire pyrolysis oil and diesel blends are unconventional fuels. Exploitation of such aspects can be a promising alternative for removing scrap tires from environment. ${ }^{3}$

Tires encompass large amounts of solid products such as carbon black $\left(\mathrm{CB}_{\mathrm{L}}\right)$ and some inorganic compounds originated from their manufacture. $\mathrm{CB}_{\mathrm{L}}$ is an amorphous material used as reinforcing filler and pigment in plastic and rubber production. From the pyrolyzed product derived from carbon black, it is also possible to obtain interesting carbonbased nanomaterials, such as activated carbon, fullerenes, 
carbon nanotubes, carbon nanoparticles, nanodiamonds, graphene oxide, graphite, among others. Such carbon-based nanomaterials can be applied in several processes, especially in the production of adsorbents, high-flux membranes, depth filters, nanocomposites, antimicrobial agents, environmental sensors, as well as in advanced technologies for controlled release of drugs in tissues, renewable energy, and in pollution prevention strategies. ${ }^{4-7}$

It is also possible to highlight the attainment of carbon-based dots (CBDs) from carbon black. These are mainly composed of graphene quantum dots and carbon quantum dots, which are in evidence among the nanocarbon materials. They are fluorescent carbon nanoparticles generally measuring 2-10 nm, presenting optical, fluorescent, and electronic properties directly related to their size. They also have several applications, e.g., in displays and lighting, photovoltaic devices, semiconductors, production of catalysts, biological markers, and imaging of tissues. ${ }^{8-11}$

The interest in quantum dots, i.e., very small nanoparticles exhibiting quantum confinement effects, leads to functional and structured properties which are not available from discrete molecules and bulk materials. Thus, the CBDs can be classified in two large groups of materials based on carbon: ${ }^{12-16}$ carbon quantum dots (CQDs), which present a quantum confinement and crystalline structure in spherical shape, and graphene quantum dots (GQDs) a not well-defined single sheet material, both exhibiting strong emissions in the visible.

Graphene and such fluorescent carbon materials $(\mathrm{CBDs})^{17}$ were introduced as a member of carbon family in $2004 .{ }^{18}$ These CBDs were synthesized in large quantities in 2006 by Sun's group, ${ }^{19}$ and there are several bottom-up ${ }^{20-25}$ preparation methods by carbonization of organic raw materials developed, as well as top-down techniques through breakdown in bulk of carbon sources. ${ }^{26-29}$ Both approaches result in CBDs capable of substituting the conventional type II-VI semiconductors quantum dots in the field of light emission materials. ${ }^{30}$ However, several synthesis methods involve additional steps of surface passivation, high temperatures, expensive raw materials, and long reaction times. For this reason, extraction techniques have been recently proposed to obtain CBDs from foods or natural materials, such as coffee, beer, humic substances, and beverages, ${ }^{31-33}$ including commercial carbon black. ${ }^{34}$

Taking these applications into account, several advantages can be explored, mainly bright fluorescence, high photostability, low toxicity, chemiluminescence, electrochemiluminescence, excellent biocompatibility, conductivity, and resistance to metabolic degradation when applied to bioprocesses. ${ }^{8,35-37}$ However, the physical, chemical, and morphological properties of carbon-based materials are extremely important for the development of real applications. ${ }^{7}$ In this context, a physicochemical method for preparing CBDs obtained from ordinary carbon black $\left(\mathrm{CB}_{\mathrm{L}}\right)$ of the pyrolytic waste tire is presented in this work.

\section{Experimental}

\section{Materials}

Analytical grade reagents were used to prepare all solutions. Sulfuric acid (98 wt.\%) was acquired from Vetec, São Paulo, Brazil. Phosphoric acid (85 wt.\%), hydrochloric acid (37 wt.\%), diethyl ether (98 wt.\%), and potassium permanganate (>99 wt.\%) were purchased from Dinâmica, Indaiatuba, Brazil. Nitric acid (65 wt.\%) and hydrogen peroxide (30 wt.\%) were acquired from Merck, São Paulo, Brazil, and sodium hydroxide (> 97 wt.\%) from Neon, Suzano, Brazil. Hydrofluoric acid (40\%) was acquired from Merck, São Paulo, Brazil.

\section{Methods}

\section{$\mathrm{CB}_{\mathrm{L}}$ purification from demineralization and oxidation} processes

The $\mathrm{CB}_{\mathrm{L}}$ was subjected to a leaching process in order to reduce minerals such as ash, sulfur, and silica concentration. For this, $42 \mathrm{~g}$ of $\mathrm{CB}_{\mathrm{L}}$ were suspended into $840 \mathrm{~mL}$ of a mixture of $\mathrm{HNO}_{3}$ and $\mathrm{HCl}(1: 1 \mathrm{v} / \mathrm{v})$ and heated to reflux temperatures for $30 \mathrm{~min}$ and then cooled to room temperature and filtered. The solid was washed with water up to get a neutral $\mathrm{pH}$ and dried at $60{ }^{\circ} \mathrm{C}$. The resulting solid was denominated $\mathrm{CB}_{\mathrm{L} 1} \cdot{ }^{38}$

Afterwards, $40 \mathrm{~g}$ of $\mathrm{CB}_{\mathrm{L} 1}$ were added to $1.4 \mathrm{~L}$ of a mixture of sulfuric acid and phosphoric acid (9:1 v/v) in a round bottom flask under mechanical stirring, and $63 \mathrm{~g}$ of potassium permanganate were slowly added in a $10 \mathrm{~mL}$ of $40 \mathrm{wt} . \%$ hydrofluoric acid solution. The reaction mixture was heated to $50{ }^{\circ} \mathrm{C}$ for $24 \mathrm{~h}$ under stirring, cooled down to room temperature and transferred to a recipient containing $1 \mathrm{~L}$ of deionized ice water.

Finally, $78 \mathrm{~mL}$ of $30 \%$ hydrogen peroxide were added, gradually. ${ }^{39}$ The mixture was filtered, and the solid obtained submitted to a continuous extraction with diethyl ether in a Soxhlet for $12 \mathrm{~h}$. The aqueous phase was filtered twice through a 0.45 -micron membrane to obtain $\mathrm{CB}_{\mathrm{L} 2}$.

\section{CBDs production from purified $\mathrm{CB}_{\mathrm{L} 2}$}

In this stage, $50 \mathrm{~mL}$ of the $\mathrm{CB}_{\mathrm{L} 2}$ suspension was put into an Erlenmeyer and reacted with sufficient $\mathrm{NaOH}$ to 
increase the $\mathrm{pH}$ to 5.6, precipitating a white fluorescent solid. This process was monitored using an UV lamp. The supernatant was centrifuged in an ultrafiltration centrifugal tube $(\mathrm{MWCO} 3 \mathrm{~K})$ at 5,000 rpm for $10 \mathrm{~min}$. Finally, the supernatant was put into a rotary evaporator $\left(60{ }^{\circ} \mathrm{C}\right.$, low pressure, $20 \mathrm{~min}$ ) yielding a pale yellow solid (CBD), which was used in all studies described now on.

\section{Characterization}

\section{UV-Vis absorption and photoluminescent emission}

UV-Vis absorption spectra of CBD dispersion in aqueous media were recorded on a Hewlett Packard 8453A diode array spectrophotometer, in the 190 to $1100 \mathrm{~nm}$ range, using a quartz cuvette. Photoluminescent emission was recorded on a Photon Technology Inc. equipment, using an InGaAs detector and Felix GX 1317 software. The analyses were performed in the 320 to $380 \mathrm{~nm}$ wavelength range.

\section{Scanning electron microcopy (SEM)}

SEM images were obtained on a high-resolution JSM 7500F (JEOL) microscope, using an acceleration voltage of $2 \mathrm{kV}$. The samples were drop casted on silicon substrate.

\section{High-resolution transmission electron microscopy (HRTEM)}

The fine particles morphology was analyzed by HRTEM using a JEOL, model JEM 2100 equipment, operating with a $\mathrm{LaB}_{6}$ electron emitting filament, at a maximum acceleration voltage of $200 \mathrm{kV}$ by means of a drop casting technique. The statistical distribution was made by the ImageJ software $1.51 \mathrm{j} 8^{40}$ only considering the spherical CBDs.

\section{Thermogravimetric analysis (TGA)}

TGA was carried out using a Shimadzu DTG 60 instrument from room temperature up to $1,000{ }^{\circ} \mathrm{C}$, at a heating rate of $10{ }^{\circ} \mathrm{C} \mathrm{min}^{-1}$, under a synthetic air atmosphere. The measurements were carried out using two platinum pans, one to place the sample and another for reference (empty).

\section{X-ray fluorescence spectra (EDX)}

The EDX were obtained at $25^{\circ} \mathrm{C}$ in a Shimadzu EDX-720 equipment, with a $\mathrm{Rh}$ tube as radiation source, setting the voltage to $15-50 \mathrm{kV}$, and current varying from 1 to $1,000 \mu \mathrm{A}$, and a $\mathrm{Si}(\mathrm{Li})$ semiconductor detector cooled in liquid nitrogen. The powder samples were placed onto Mylar $^{\circledR}$ films in a sample holder with $30 \mathrm{~mm}$ of diameter and analyzed.

\section{Infrared microscopy ( $\mu \mathrm{FTIR})$}

The CBDs material were collected, rinsed with nanopure water and allowed to dry on a rotary evaporator at $60{ }^{\circ} \mathrm{C}$. The dried sample was monitored on a Shimadzu AM30 $\mu$ FTIR microscope, and the vibrational spectrum collected directly from the powder, scanning from 800 to $4000 \mathrm{~cm}^{-1}$ and collecting the average of 32 scans per point.

\section{X-ray photoelectron spectroscopy (XPS)}

A Thermo-Fisher Scientific XPS device, K-alpha+ model, was used to obtain the XPS spectrum. The equipment analyzed solid, powdered or densified samples, allowing the identification of chemical elements (except $\mathrm{He}$ and $\mathrm{H}$ ) and their oxidation states on the surface of materials. The radiation penetration was $10 \mathrm{~nm}$. The equipment has an argon ion beam for load compensation and performs experiments as a function of depth profile.

\section{X-ray diffractometry (XRD)}

The crystalline structures of samples were surveyed by XRD investigation on D2 Phaser diffractometer from Bruker company with $\mathrm{Cu} \operatorname{K} \alpha$ radiation $(\lambda=1.5418 \AA)$ at

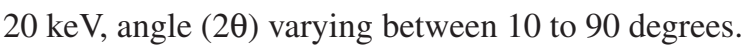

\section{Results and Discussion}

A physicochemical method to obtain CBDs from carbon black $\left(\mathrm{CB}_{\mathrm{L}}\right)$ derived from tires is shown in Figure 1. Two processes were possible to obtain the CBDs, one was the direct solvent extraction and other with our methodology.

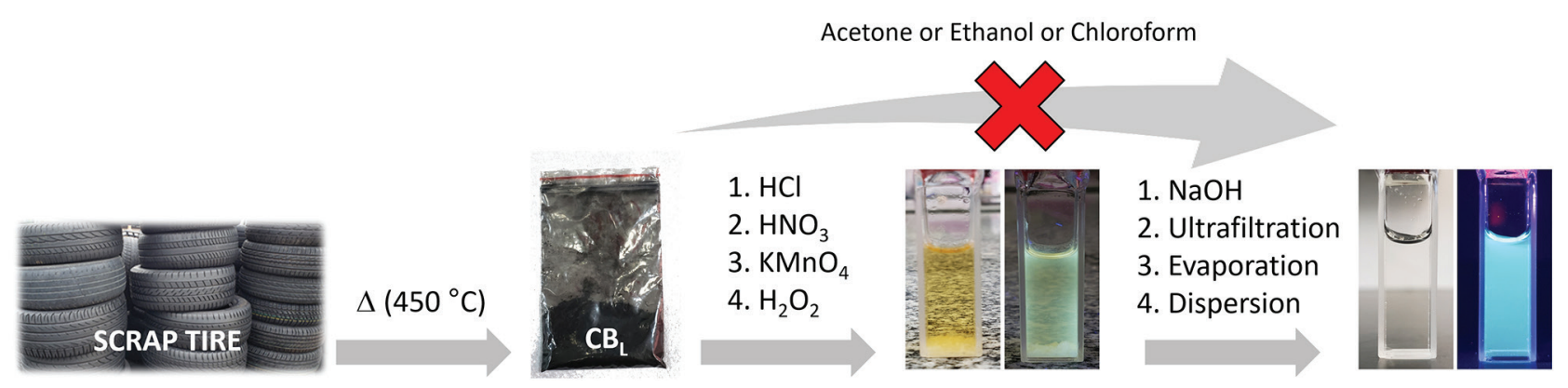

Figure 1. Schematic illustration of the process to obtain purified CBDs from waste tire, highlighting the importance of all stages independently of the origin of tires. 
The characteristics of the CBD particles shown in this article, revealed that the purification method is critical and must be applied to ensure the elimination of impurities which may alter the photoluminescence (PL). For this reason, it is not recommended the use of a physical method or any organic solvent in a direct extraction process. Due to varying tire sources, various impurities, molecules or materials that may be carried along the direct extraction process of carbon black from tire pyrolysis, especially without prior chemical treatment or using acetone as the extraction solvent, for example. This supernatant can be rotoevaporated and the solid obtained as $\mathrm{CBD}$, can be resuspended again in another organic solvent to obtain a comparative PL spectra. A not so well-defined emission profile was observed, contrary to the case with the CBDs obtained by our purification method. Therefore, the presence of impurities in direct extraction can decrease the quantum emission yield, presumably through trapping states, altering its emission profile (PL), leading to wide bands.

A carbon black purification process was carried out by a demineralization stage to partially remove $\mathrm{S}$ and $\mathrm{Si}$, as well as decrease the amount of $\mathrm{Zn}$ and ashes in $\mathrm{CB}_{\mathrm{L} 1}$, resulting from the tire manufacturing and vulcanization. An oxidation process was also performed to obtain $\mathrm{CB}_{\mathrm{L} 2}$. Purified $\mathrm{CB}_{\mathrm{L}}$ was obtained after these processes and characterized by DRX as shown in Figure 2.

The fluorescence of the residual suspension of the $\mathrm{CB}_{\mathrm{L} 2}$ purification process indicates the presence of $\mathrm{CBDs}$, which was observed as an intense green fluorescence from the light-yellow colored suspension when irradiated with a blue laser pointer. Afterwards, this suspension was precipitated with sodium hydroxide, separated, ultracentrifuged and the supernatant was rotoevaporated, and their physicochemical properties evaluated. Fluorescence spectroscopy experiments were performed to characterize this material.
One of the characteristics of carbon-based dots materials is the excitation wavelength-dependent photoluminescence (PL), resulting in varied emission wavelengths. These materials present a contrasting behavior as compared to common luminescent organic materials, whose characteristic emitting state is unique and presents lower energy, i.e., a specific emission wavelength.

The CBDs were light yellow under visible illumination, while they emitted bright blue fluorescence under UV-Vis light excitation under $365 \mathrm{~nm}$. The CBDs displayed a strong UV-Vis absorption peak located at $247 \mathrm{~nm}$, which is typically assigned to the $\pi-\pi^{*}$ transition of $\operatorname{sp}^{2}$ domains from carbon materials. ${ }^{41,42}$ The phenomenon of excitation wavelength-dependent emission is clearly observed in Figure 3. It was possible to see that the emission spectra were sensitive to the excitation wavelength. The emission peak varied from 380 to $495 \mathrm{~nm}$ as the excitation wavelength increased from 320 to $380 \mathrm{~nm}$, showing a strong red-shift as mentioned in previous studies. ${ }^{43}$

The PL spectra described in Figure 3 shows CBDs exhibiting a strong PL peak at $415 \mathrm{~nm}$ at the maximum excitation wavelength. The CBDs' quantum yield was approximately $19.29 \%$ (see the Supplementary Information section). The CBDs' PL gradually decreased within fifteen days because of the agglomeration process and ageing. In addition, the photos of colloidal suspensions of CBDs' prepared soon after the oxidation procedure under daylight (left) and UV lamp irradiation at $365 \mathrm{~nm}$ (right) are shown in the inset of Figure 3a.

The HRTEM micrographs are shown in Figure 4. Both micrographs show that CBDs consist of very small particles with not well-defined shape. In calculating size distribution, the spherical shape of the CBD was used as criteria. Both morphological structure characteristics are consistent with what is reported for these new carbon-based nanomaterials, where the size corresponds to a value below $10 \mathrm{~nm}^{44,45}$

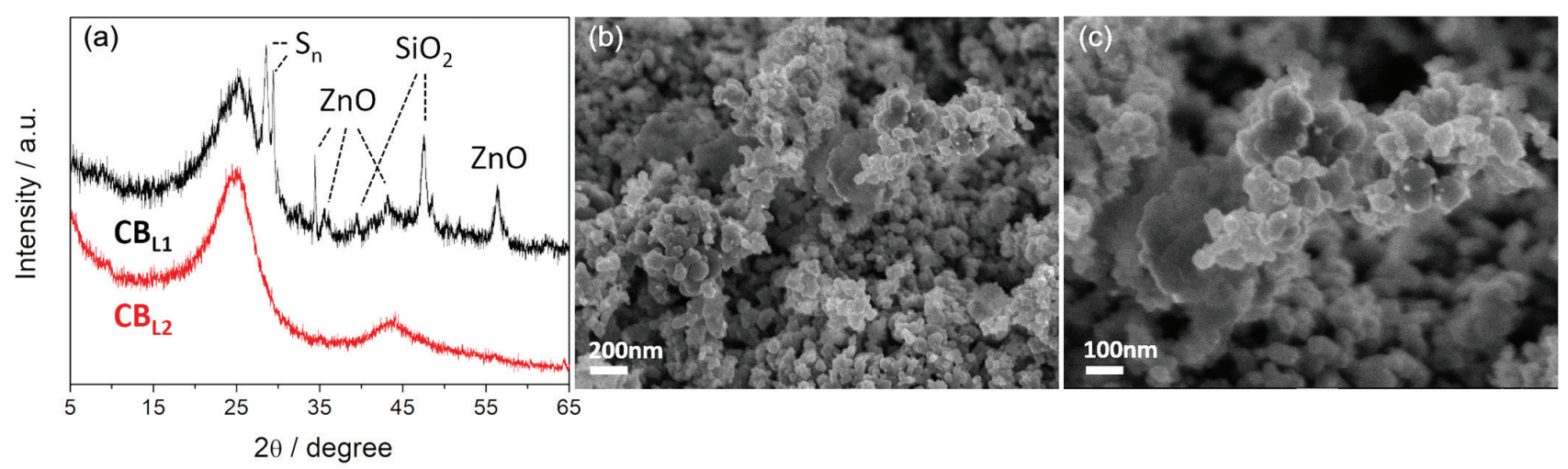

Figure 2. (a) XRD analysis showing the efficiency of the purification method in removing impurities, leading to high luminescent CBDs. (b) and (c) SEM images evidencing the non-homogeneous shape and structure of the $\mathrm{CB}_{\mathrm{L} 2}$. 

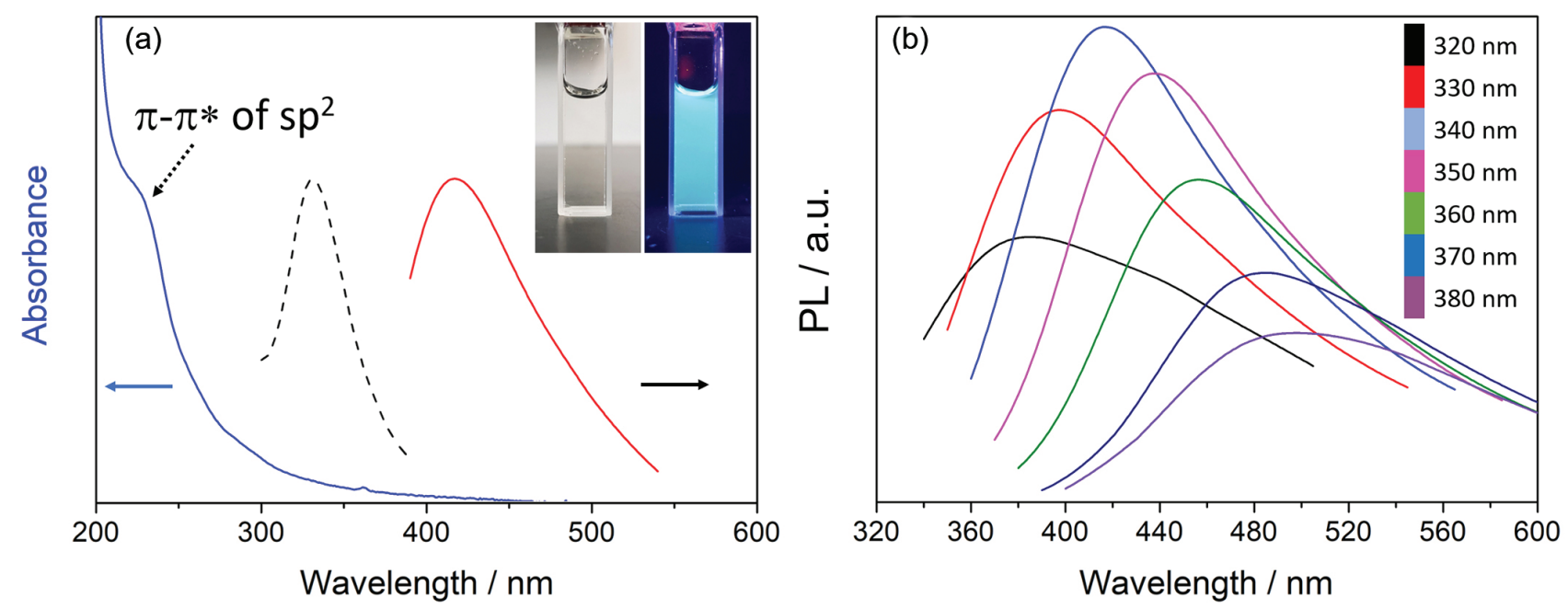

Figure 3. (a) UV-Vis absorption (blue line), maximum PL excitation wavelength (dotted line), maximum PL emission wavelength (red line). Inset: photo of a CBD suspension under daylight (left) and UV lamp irradiation at $365 \mathrm{~nm}$ (right). (b) The emission spectra from 320 to $380 \mathrm{~nm}$ demonstrating the dependence on the excitation wavelength.
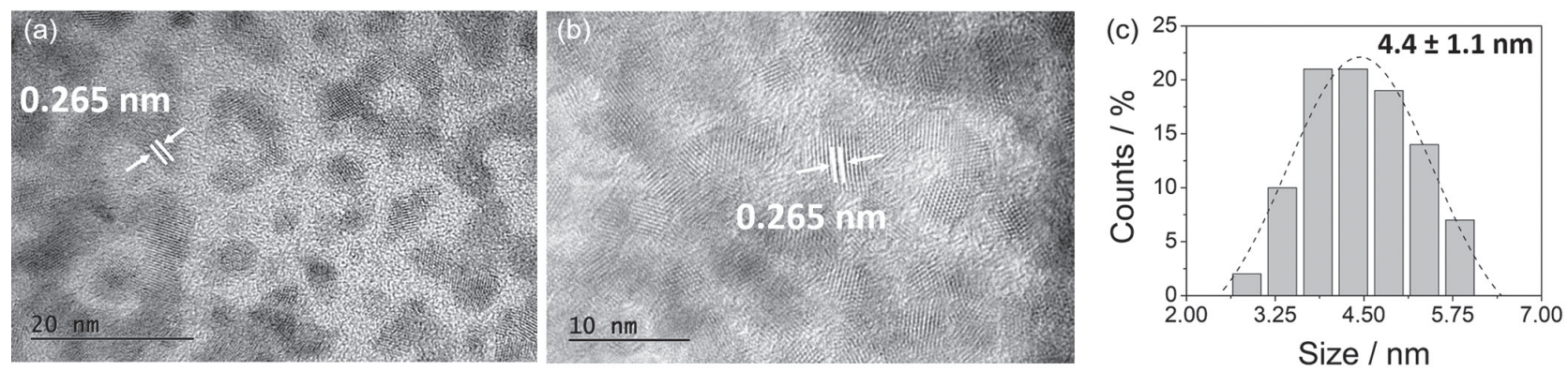

Figure 4. (a, b) HRTEM micrographs of CBD particles with not well-defined shape; (c) size distribution histogram of the spherical structures.

The CBD particle dispersion was not mostly spherical shaped and monodisperse in our specific case as observed in HRTEM, contrary to the literature. ${ }^{45}$ It is common to observe aggregates according to the sample preparation by drop casting method, as reporting by Ray et al. ${ }^{46}$ in 2009. The images reflect a closer characteristic of GQD than the CQD one. ${ }^{47}$ However, two types of CBDs may commonly be originated from the pyrolysis of organic molecules submitted to high temperatures. ${ }^{48}$ One structure is related to CQD, a quasi-spherical nanoparticle consisting of amorphous and crystalline parts. The second structure, GQDs, usually have graphene lattices inside the dots, resembling the crystalline structure of single or few layered graphene. It is relevant to note that the PL emission of this type of material happens when electron trapping states are present in the bandgap, due to superficial imperfections or functional groups on the surface. In this case, the recombination of the electron-hole pair leads to a greater radioactive wavelength emission.

The absorption and emission results indicate that the PL response of CBD material is probably a specific combination of the intrinsic quantization effect of the core and the particle's surface, which is ruled by the surface functional groups. In this study, due to the oxidation process, several carboxylic groups produced by the oxidation process appear on the surface, as it is shown by FTIR spectroscopy and X-ray photoelectron spectroscopy (XPS) (Figure 5). The oxidation must increase the occurrence of trapping states and wavelength dependent excitation emission.

In order to identify the surface groups of CBDs, the materials were investigated by FTIR and XPS analyses. There is a broad band at about $3,400 \mathrm{~cm}^{-1}$ signed to hydrophilic groups such as the $\mathrm{O}-\mathrm{H}$ and/or $\mathrm{N}-\mathrm{H}$ stretching on the surface, as well as $\mathrm{C}-\mathrm{H}$ stretching bands at about $2,930 \mathrm{~cm}^{-1}$ and $2,850 \mathrm{~cm}^{-1}$. Moreover, the band at about $1,740 \mathrm{~cm}^{-1}$ indicates the presence of carbonyl $(\mathrm{C}=\mathrm{O})$ groups, and the one at about $1,600 \mathrm{~cm}^{-1}$ was assigned to $\mathrm{C}=\mathrm{C}$ stretching of polycyclic aromatic hydrocarbons, which is consistent with UV-Vis absorption spectra. ${ }^{43}$

There is a broad band starting from $1,480 \mathrm{~cm}^{-1}$ until $1,160 \mathrm{~cm}^{-1}$ indicating epoxide/ether $\mathrm{C}-\mathrm{O}-\mathrm{C}$ and deformation vibrations of $\mathrm{CH}_{2}$ and $\mathrm{CH}_{3}$ present on the surface. These last bands at about 1,090, 980, and $880 \mathrm{~cm}^{-1}$ can be signed as $\mathrm{C}-\mathrm{H}$ bending, ring puckering and $\mathrm{N}-\mathrm{H}$ 

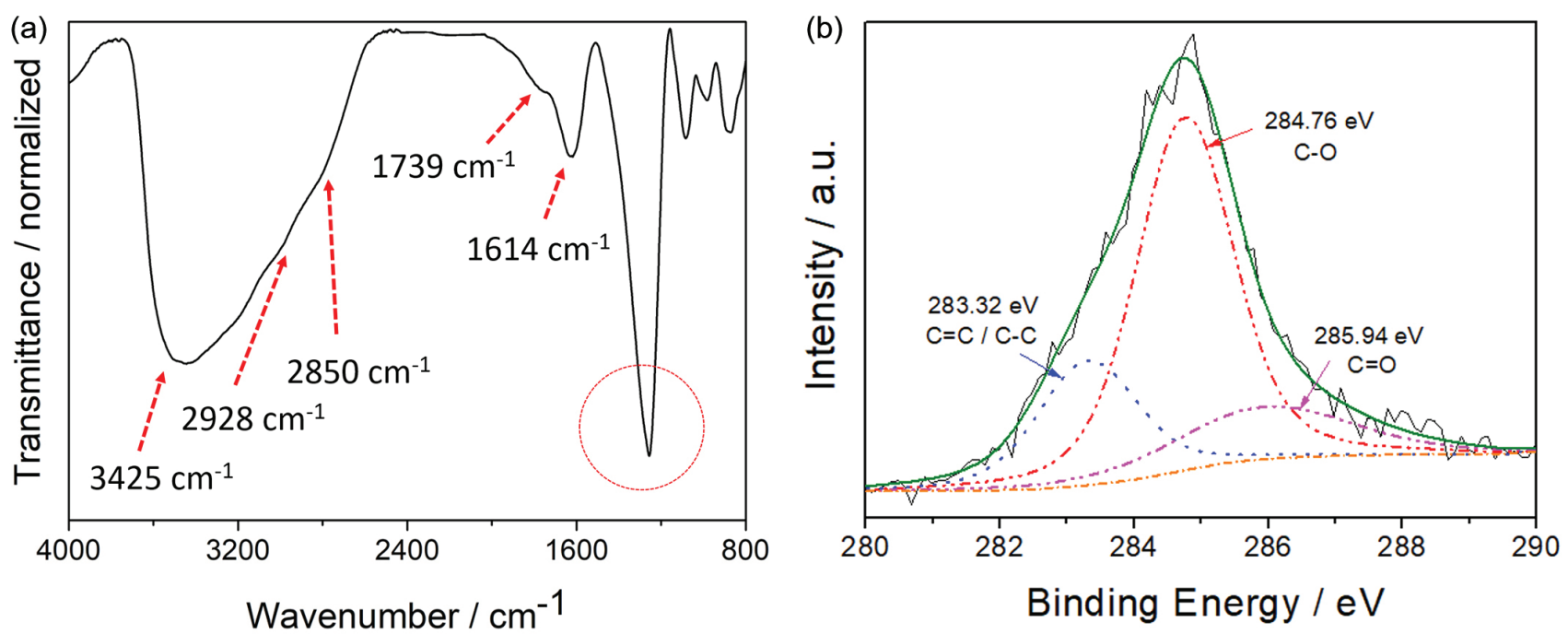

Figure 5. (a) FTIR microscopy spectrum of the purified CBDs showing the characteristic vibrational peaks. (b) C1s XPS spectrum of the purified CBDs that could be further deconvoluted into three peaks $\left(\mathrm{sp}^{2}, \mathrm{sp}^{3}\right.$ and carbonyl groups).

wagging. These results show that the CBDs have abundant $\mathrm{CH}_{2}$ and $\mathrm{CH}_{3}$ groups, as well as several hydrophilic groups such as carboxyl and hydroxyl groups on their surface, which can explain the PL feature of this material and the presence of GQDs and CQDs. The high resolution C1s XPS spectrum of CBDs corroborates with the FTIR results. It could be further deconvoluted into three bands, corresponding to $\mathrm{sp}^{2}(\mathrm{C}=\mathrm{C} / \mathrm{C}-\mathrm{C}, 283.32 \mathrm{eV}), \mathrm{sp}^{3}(\mathrm{C}-\mathrm{O}$, $284.76 \mathrm{eV})$, and carbonyl carbons $(\mathrm{C}=\mathrm{O}, 285.94 \mathrm{eV}) .^{12,44}$

The use of demineralization and desulfurization agents play an important role to remove ashes, silica, and sulfur as described by Banar et al..$^{37}$ Also, using oxidant agents produce a carboxyl groups and hydroxyl in CBD material, becoming soluble in water, according to assignment of FTIR and XPS of this material. The addition of $\mathrm{NaOH}$ after this process is very important to precipitate metal hydroxides which remained in the sample after treatment with $\mathrm{KMnO}_{4}$ (Figure 6).

Analyzing the TGA of solid II in Figure 6b, the absence of a substantial quantity of manganese (remaining $0.2 \%$ in weight) and zinc was verified in the purified CBDs, ${ }^{49-51}$ where no substantial mass loss was observed, only a drying process. These purified CBDs show potential as fluorescent agents in future applications, such as non-destructive tests ${ }^{52}$ and bioimaging. ${ }^{53}$

It is important to see in Figure $6 \mathrm{~b}$ that mass loss keeps occurring after $800{ }^{\circ} \mathrm{C}$ for solid I. At about $1,000{ }^{\circ} \mathrm{C}$, $\alpha-\mathrm{Mn}_{2} \mathrm{O}_{3}$ is unstable and decompose into $\mathrm{Mn}_{3} \mathrm{O}_{4}$. This temperature effect is due to the negative values of the formation entropy term, which in general is more negative
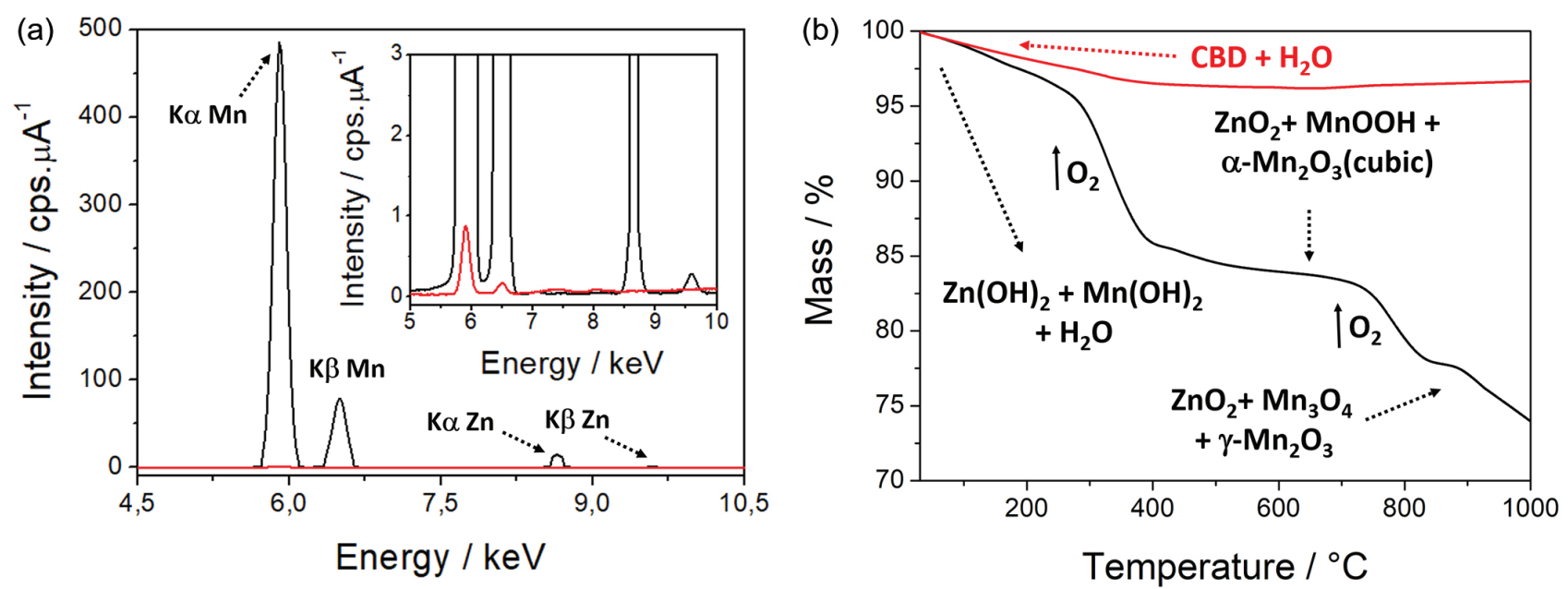

Figure 6. (a) EDX spectra of the solid obtained after the first precipitation with $\mathrm{NaOH}$ (black line-solid I) and EDX spectra of the purified CBD obtained after solvent evaporation (red line-solid II); (b) TGA of the solid obtained after the first precipitation with NaOH (black line-solid I), and TGA of the purified CBD obtained after solvent evaporation (red line-solid II). 
for materials with manganese ions in higher oxidation states. Besides, it should not be inferred that there is not $\mathrm{Mn}_{2} \mathrm{O}_{3}$ at all in the final residue under the conditions used here. Some amount of $\gamma-\mathrm{Mn}_{2} \mathrm{O}_{3}$ could still remain after this treatment. The EDX spectra in Figure 6a shown at inset, the lowering intensity signal referring to manganese and zinc elements, indicating an efficient purification process. Finally, the goals of the work were achieved as can be seen by applying a methodology to obtain a purer CBD than simply extracted directly with an organic solvent. However, it must be clear that the obtained samples are directly related to the tire source. For that reason, sometimes, the samples are not homogeneous, and its characterization became a challenge.

\section{Conclusions}

The pyrolysis of unserviceable tires has been an alternative to improper disposal of waste tire, as it may contribute to remove these residues from inappropriate locations. This study presents a physicochemical method to prepare CBDs from regular tires.

The extracted CBDs showed water solubility and presented an emission behavior dependent on the excitation wavelength and a high quantum yield of up to $19.29 \%$ compared to other carbon dots in the literature. Besides, this type of material showed a mixture of two kinds of structures: one well-defined spherical shape with confinement and crystalline structure (CQDs) and another not well-defined shape conjugated single sheet of graphene (GQDs), giving rise to a potential class of materials which may be used in fluorescence imaging and optoelectronic devices.

\section{Supplementary Information}

Supplementary information about quantum yield procedure is available free of charge at http://jbcs.sbq.org.br as PDF file.

\section{Acknowledgments}

The authors greatly acknowledge the support from São Paulo Research Foundation (FAPESP) (grants No. 2013/24725-4, 2017/09469-2), National Council for Scientific and Technological Development (CNPq) (grant No. 482383/2013-5). This study was financed in part by the Coordination of Superior Level Staff ImprovementBrazil (CAPES). The authors would like to thank Prof Dr Henrique Eisi Toma and Koiti Araki for granting access to the Supramolecular Chemistry and Nanotechnology
Laboratory (LQSN) infrastructure and UFABC multi-user center, Santo André Campus for the XPS measurements.

\section{Author Contributions}

J. B. Parra, K. C. Silva, P. A. S. Valezin, R. G. Martins, R. R. Gomes were responsible for conceptualization and investigation; R. S. Pereira for data curation; F. M. de Melo for investigation, data curation and formal analysis; A. A. Morandim-Giannetti, R. G. dos Santos, L. C. G. A. Panzarini for writing original draft and formal analysis; $M$. M. Toyama for data curation, formal analysis, investigation, project administration, writing original draft and writingreview and editing.

\section{References}

1. Martínez, J. D.; Cardona-Uribe, N.; Murillo, R.; García, T.; López, J. M.; Waste Manage. 2019, 85, 574.

2. Costa, G. A.; Santos, R. G.; Fuel 2019, 241, 558.

3. Umeki, E. R.; de Oliveira, C. F.; Torres, R. B.; dos Santos, R. G.; Fuel 2016, 185, 236.

4. Compton, O. C.; Nguyen, S. T.; Small 2010, 6, 711.

5. Peng, J.; Gao, W.; Gupta, B. K.; Liu, Z.; Romero-Aburto, R.; Ge, L.; Song, L.; Alemany, L. B.; Zhan, X.; Gao, G.; Vithayathil, S. A.; Kaipparettu, B. A.; Marti, A. A.; Hayashi, T.; Zhu, J.-J.; Ajayan, P. M.; Nano Lett. 2012, 12, 844.

6. Cha, C.; Shin, S. R.; Annabi, N.; Dokmeci, M. R.; Khademhosseini, A.; ACS Nano 2013, 7, 2891.

7. Mauter, M.; Elimelech, M.; Environ. Sci. Technol. 2008, 42, 5843.

8. Namdari, P.; Negahdari, B.; Eatemadi, A.; Biomed. Pharmacother. 2017, 87, 209.

9. Dong, Y.; Wan, L.; Cai, J.; Fang, Q.; Chi, Y.; Chen, G.; Sci. Rep. 2015, 5, 10037.

10. Wei, W.; Lu, C.; WenGuang, L.; Sci. China: Chem. 2014, 57, 522.

11. Wang, Y.; Hu, A.; J. Mater. Chem. C 2014, 2, 6921.

12. Khan, Z. M. S. H.; Rahman, R. S.; Shumaila; Islam, S.; Zulfequar, M.; Opt. Mater. 2019, 91, 386.

13. Li, X.; Rui, M.; Song, J.; Shen, Z.; Zeng, H.; Adv. Funct. Mater. 2015, 25, 4929.

14. Das, R.; Bandyopadhyay, R.; Pramanik, P.; Mater. Today Chem. 2018, 8, 96.

15. Shen, L.-M.; Liu, J.; Talanta 2016, 156, 245.

16. Zhang, J.; Yu, S.-H.; Mater. Today 2016, 19, 382.

17. Rodrigues, C. V.; Correa, J. R.; Aiube, C. M.; Andrade, L. P.; Galvão, P. M.; Costa, P. A.; Campos, A. L.; Pereira, A. J.; Ghesti, G. F.; Felix, J. F.; Weber, I. T.; Neto, B. A.; Rodrigues, M. O.; J. Braz. Chem. Soc. 2015, 26, 2623.

18. Xu, X.; Ray, R.; Gu, Y.; Ploehn, H. J.; Gearheart, L.; Raker, K.; Scrivens, W. A.; J. Am. Chem. Soc. 2004, 126, 12736. 
19. Sun, Y. P.; Zhou, B.; Lin, Y.; Wang, W.; Fernando, K. A. S.; Pathak, P.; Meziani, M. J.; Harruff, B. A.; Wang, X.; Wang, H.; Luo, P. G.; Yang, H.; Kose, M. E.; Chen, B.; Veca, L. M.; Xie, S.-Y.; J. Am. Chem. Soc. 2006, 128, 7756.

20. Wang, J.; Wang, C.-F.; Chen, S.; Angew. Chem., Int. Ed. 2012, 124, 9431.

21. Zhai, X.; Zhang, P.; Liu, C.; Bai, T.; Li, W.; Dai, L.; Liu, W.; Chem. Commun. 2012, 48, 7955.

22. Bourlinos, A. B.; Stassinopoulos, A.; Anglos, D.; Zboril, R.; Karakassides, M.; Giannelis, E. P.; Small 2008, 4, 455.

23. Kozák, O.; Sudolská, M.; Pramanik, G.; Cígler, P.; Otyepka, M.; Zbořil, R.; Chem. Mater. 2016, 28, 4085.

24. Martindale, B. C.; Hutton, G. A.; Caputo, C. A.; Reisner, E.; J. Am. Chem. Soc. 2015, 137, 6018.

25. Sun, D.; Ban, R.; Zhang, P.-H.; Wu, G.-H.; Zhang, J.-R.; Zhu, J.-J.; Carbon 2013, 64, 424.

26. Zhou, J.; Booker, C.; Li, R.; Zhou, X.; Sham, T.-K.; Sun, X.; Ding, Z.; J. Am. Chem. Soc. 2007, 29, 744.

27. Canevari, T. C.; Nakamura, M.; Cincotto, F. H.; Melo, F. M.; Toma, H. E.; Electrochim. Acta 2016, 209, 464.

28. Liu, H.; Ye, T.; Mao, C.; Angew. Chem., Int. Ed. 2007, 46, 6473.

29. Zhang, X.; Wang, Y. Z.; Kalytchuk, S.; Kershaw, S. V.; Wang, Y.; Wang, P.; Zhang, T.; Zhao, Y.; Zhang, H.; Cui, T.; Wang, Y.; Zhao, J.; Yu, W. W.; Rogach, A. L.; ACS Nano 2013, 7, 11234.

30. Jiang, C.; Wu, H.; Song, X.; Ma, X.; Wang, J.; Tan, M.; Talanta 2014, 127, 68 .

31. Wang, Z.; Liao, H.; Wu, H.; Wang, B.; Zhao, H.; Tan, M.; Anal. Methods 2015, 7, 8911.

32. Liao, H.; Jiang, C.; Liu, W.; Vera, J. M.; Seni, O. D.; Demera, K.; Yu, C.; Tan, M.; J. Agric. Food Chem. 2015, 63, 8527.

33. Dong, Y.; Chen, C.; Zheng, X.; Gao, L.; Cui, Z.; Yang, H.; Guo, C.; Chi, Y.; Li, C. M.; J. Mat. Chem. 2012, 22, 8764.

34. Luo, P. G.; Yang, F.; Yang, S.-T.; Sonkar, S. K.; Yang, L.; Broglie, J. J.; Liu, Y.; Sun, Y.-P.; RSC Adv. 2014, 4, 10791.

35. Dong, Y.; Pang, H.; Yang, H. B.; Guo, C.; Shao, J.; Chi, Y.; Li, C. M.; Yu, T.; Angew. Chem. 2013, 125, 7954.

36. Anilkumar, P.; Wang, X.; Cao, L.; Sahu, S.; Liu, J.-H.; Wang, P.; Korch, K.; Tackett II, K. N.; Parenzan, A.; Sun, Y.-P.; Nanoscale 2011, 3, 2023.
37. Banar, M.; Ozkan, A.; Akyildiz, V.; Çokaygil, Z; Onay, O.; J. Mater. Cycles Waste Manage. 2015, 17, 125.

38. Marcano, D. C.; Kosynkin, D. V.; Berlin, J. M.; Sinitskii, A.; Sun, Z.; Slesarev, A.; Alemany, L. B.; Wei Lu; Tour, J. M.; ACS Nano 2010, 4, 4806.

39. Arcud, F.; Dord-evic', L.; Prato, M.; Angew. Chem., Int. Ed. 2016, 55, 2107.

40. Rasband, W. S.; ImageJ, version 1.51j8; U. S. National Institutes of Health, Bethesda, Maryland, USA, 2017.

41. Ding, H.; Yu, S. B.; Wei, J. S.; Xiong, H. M.; ACS Nano 2016, $10,484$.

42. Zhu, H.; Wang, X.; Li, Y.; Wang, Z.; Yang, F.; Yang, X.; Chem. Commun. 2009, 34, 5118.

43. Pires, N. R.; Santos, C. M. W.; Sousa, R. R.; de Paula, R. C. M.; Cunha, P. L. R.; Feitosa, J. P. A.; J. Braz. Chem. Soc. 2015, 26, 1274 .

44. Wang, C.; Shang, S.; Zheng, X.; Lei, P.; Han, J.; Yuan, L.; Li, Z.; Wang, R.; Gong, W.; Tang, J.; Yang, Y.; J. Braz. Chem. Soc. 2019, 30, 988.

45. Cayuela, A.; Soriano, M. L.; Carrión, C. C.; Valcárcel, M.; Chem. Commun. 2016, 52, 1311.

46. Ray, S. C.; Saha, A.; Jana, N. R.; Sarkar, R.; J. Phys. Chem. C 2009, 113, 18546.

47. Pan, D.; Zhang, J.; Li, Z.; Wu, M.; Adv. Mater. 2010, 22, 734.

48. Zheng, X. T.; Ananthanarayanan, A.; Luo, K. Q.; Chen, P.; Small 2015, 11, 1620.

49. Terayama, K.; Ikeda, M.; Trans. Jpn. Inst. Met. 1983, $24,754$.

50. Chen, W.; Lu, Y. H.; Wang, M.; Kroner, L.; Paul, H.; Fecht, H.-J.; Bednarcik, J.; Stahl, K.; Zhang, Z. L.; Wiedwald, U.; Kaiser, U.; Ziemann, P.; Kikegawa, T.; Wu, O. C. D.; Jiang, J. Z.; J. Phys. Chem. 2009, 113, 1320.

51. Melo, F. M.; Grasseschi, D.; Brandão, B. B. N. S.; Fu, Y.; Toma, H. E.; ACS Appl. Nano Mater. 2018, 1, 2858.

52. Oliveira, D.; Melo, F. M.; Toma, H. E.; Micron 2018, 108, 19.

53. Nosrollahi, F.; Koh, Y. R.; Chen, P.; Varshosaz, J.; Khodadadi, A. A.; Lim, S.; Mater. Sci. Eng. Carbon 2019, 94, 247.

Submitted: January 15, 2020 Published online: April 17, 2020 